\title{
Os Cuidados Paliativos no Ensino Médico Pré-Graduado: Perspectivas dos Estudantes Finalistas De Medicina e dos Internos de Formação
} Geral

\author{
Palliative Care and Pre-Graduate Medical Education: Perspectives of \\ Last-Year Medical Students and Medical Interns
}

Pedro Frazão' (https://orcid.org/0000-0002-9132-4363) Paulo Reis-Pina ${ }^{2,3}$ (https://orcid.org/0000-0002-4665-585X)

\section{Resumo:}

Introdução: Em Portugal, o envelhecimento populacional e a elevada prevalência de doenças crónicas justificam o investimento na educação dos profissionais de saúde em cuidados paliativos (CP). Este estudo teve como objectivo principal avaliar o ensino pré-graduado de CP e o seu contributo para a profissão médica, na percepção dos alunos do curso de Medicina e dos internos recém-formados.

Material e Métodos: Estudo qualitativo, com questionário electrónico e tratamento descritivo dos dados, cujos participantes foram alunos finalistas das Escolas Médicas de Portugal e médicos internos de formação geral.

Resultados: Dos 221 participantes (61\% estudantes), a maioria teve aulas de CP, com duração $<8$ horas. Os inquiridos consideraram pertinente a disciplina de CP na educação médica, mas esta deveria ter maior carga horária e deveria conceder os conhecimentos necessários para qualquer médico generalista. Deveria existir mais ensino sobre os sintomas dos doentes terminais, os sinais físicos de morte iminente e a comunicação de más notícias. Os inquiridos revelaram um bom conhecimento sobre a filosofia dos CP e sentiam-se familiarizados com os principais conceitos dos CP. Os estudantes não tinham acesso a um ambiente prático, especializado em CP.

Conclusão: Em Portugal, embora o ensino pré-graduado em CP seja insuficiente, tem tido um efeito positivo, com elevados conhecimentos teóricos, mas com pouca prática em CP. As Escolas Médicas devem investir mais no ensino pré-graduado de CP, através do currículo obrigatório e da prática regular (contacto clínico), capacitando os estudantes para uma relação, empática e compassiva, entre o médico e o doente em CP.

Palavras-chave: Conhecimentos, Atitudes e Prática em Saúde; Cuidados Paliativos; Educação Médica; Estudantes de Medicina; Internato e Residência.

${ }^{1}$ Medicina Interna, Hospital Ortopédico Sant'iago do Outão, Centro Hospitalar de Setúbal, Setúbal, Portugal

"Unidade de Cuidados Paliativos "Bento Menni", Casa de Saúde da Idanha, Sintra, Portugal

${ }^{3}$ Faculdade de Medicina da Universidade de Lisboa, Lisboa,

Portugal

DOI: 10.24950/O/166/20/1/2021

\section{Abstract:}

Introduction: In Portugal, the ageing of the population, and the high prevalence of chronic diseases justify the investment in the education of health professionals in palliative care (PC). The main objective of this study was to assess the opinions of medical students and interns about pre-graduate education in $P C$, and its contribution to the medical profession.

Material and Methods: Qualitative study (online questionnaire) with descriptive treatment of the data. Participants were last-year students at Medical Schools of Portugal and first-year interns.

Results: Of the 221 participants, 61\% were students, the majority attended PC classes which lasted $<8$ hours. Participants considered the discipline of $P C$ relevant for the medical education, but it should have a higher workload and should give the necessary knowledge to any general practitioner. There should be more education on symptoms of terminally ill patients, physical signs of imminent death, and communication of bad news. Participants revealed a good knowledge of the philosophy of PC and felt familiar with the main concepts of PC. Students did not have access to a practical, specialized environment for $P C$.

Conclusion: In Portugal, although the pre-graduate education in PC is insufficient, it has had a positive effect, with high theoretical knowledge, but with little practice in PC. Medical Schools should invest more in pre-graduate education in PC, through the obligatory curriculum and regular clinical practices, empowering students for an empathic and compassionate relationship between the doctor and the patient in PC.

Keywords: Health Knowledge, Attitudes, Practice; Internship and Residency; Medical Education; Palliative Care; Students, Medical.

\section{Introdução}

Os cuidados paliativos (CP) tornaram-se ao longo das últimas décadas um bem necessário para toda a população. Considerados um direito humano, os CP são transversais a diversos contextos de saúde, desde os cuidados primários aos terciários. ${ }^{1,2}$ 
O desempenho cada vez mais relevante dos CP na sociedade hodierna pode ser explicado, quer pelo aumento da esperança média de vida, quer pelo acréscimo de doenças crónicas e comorbilidades. ${ }^{3,4}$ Neste sentido, várias organizações internacionais, como o Conselho da Europa, e nacionais, como a Comissão Nacional de Cuidados Paliativos, recomendam o desenvolvimento de estratégias que promovam a educação em CP.5,6

A Organização Mundial de Saúde identifica como barreira à prestação de CP a formação limitada ou inexistente dos profissionais de saúde neste domínio, o que condiciona a resposta eficiente às necessidades paliativas dos doentes. ${ }^{3}$ Nos diversos cursos da área da saúde, a maioria dos estudantes não detém conhecimentos suficientes sobre CP, não possuindo capacitação para prestar cuidados adequados à população necessitada. ${ }^{7}$

Em Portugal, a formação dos profissionais em CP constitui uma limitação ao alargamento de serviços. ${ }^{5,8}$ De facto, a formação e a investigação são componentes de um eixo estratégico para o desenvolvimento nacional dos CP. ${ }^{9}$ Pretende-se fomentar a formação pré-graduada em CP, com prática clínica, nos planos curriculares de todas as escolas de medicina, enfermagem, psicologia e serviço social. ${ }^{9}$

Desde o ano letivo 2016/2017 que se incluiu o ensino de CP no Mestrado Integrado em Medicina, em Portugal, sob a forma de currículo obrigatório. Passados dois anos letivos, o objetivo deste trabalho é analisar o ensino de CP na formação pré-graduada nas oito escolas médicas (EM) portuguesas, através da perspetiva dos estudantes finalistas do Mestrado Integrado em Medicina e dos Internos de Formação Geral (IFG) do ano civil 2019 que tinham frequentado uma EM nacional. Ademais, pretende-se avaliar alguns conhecimentos fundamentais em CP e perceber a influência da educação em $\mathrm{CP}$ na futura vida profissional.

\section{Material e Métodos}

Foi elaborado um questionário eletrónico através da plataforma Google Docs ${ }^{\circledR}$, construído com base em investigações portuguesas decorridas noutros contextos, ${ }^{19}$ e em estudos semelhantes realizados noutros países.10-12 O inquérito versou sobre:

i) contacto com os CP e a formação pré-graduada em medicina paliativa (cinco questões);

ii) avaliação sobre o ensino de CP e a sua importância (onze questões); e

iii) aferição de algumas noções fundamentais sobre os CP (doze questões). Foram apresentadas algumas afirmações (de A1 a A23) e questionado o grau de concordância dos inquiridos com as mesmas, segundo uma escala tipo Likert contendo seis posicionamentos, desde o "Discordo" (totalmente, bastante, pouco) ao "Concordo" (pouco, bastante, totalmente).

Foi aplicado a estudantes do ano letivo 2018/2019 (do
Mestrado Integrado em Medicina das oito EM do país) e a IFG do ano civil de 2019 (que tenham frequentado uma EM portuguesa nos dois anteriores). A escolha destes últimos deveu-se ao facto de estarem no mercado de trabalho e terem podido frequentar as aulas de CP durante o curso médico, ao abrigo do currículo opcional ou obrigatório (o último surgido em 2016/2017), dependendo da EM frequentada.

Para operacionalizar o estudo foram contactadas as Direcções das oito EM e solicitada a divulgação do questionário junto das Associações de Estudantes das EM. No caso dos IFG, o inquérito foi divulgado através da rede social Facebook $^{\circledR}$, num grupo que reunia vários IFG do país.

A resposta ao inquérito era voluntária, sendo assegurado o anonimato durante todo o estudo. Foram apenas recolhidos dados sobre: género, idade e a EM onde o inquirido ainda estudava ou tinha estudado.

O estudo decorreu de 25 de fevereiro a 06 de abril de 2019.

\section{Resultados}

As respostas foram analisadas em função de tendências inequívocas, no sentido da concordância ou discordância, sofrendo uma análise qualitativa e descritiva.

Em todas as questões foram utilizadas as estatísticas n e percentagem (\%) para expressar de forma absoluta e relativa, respetivamente, a quantidade de respostas obtidas em cada posicionamento.

\section{COMPONENTE DEMOGRÁFICA}

Houve 221 respostas, efetuadas por 168 mulheres (76\%), sendo 134 estudantes (61\%). A idade (anos) teve: média = 25,36, desvio-padrão = 3,39, mínimo de 22 e máximo de 40 .

A distribuição das respostas foi, por EM: Faculdade de Medicina da Universidade de (Lisboa - 45,0\%, Coimbra 12,2\%, Porto - 2,2\%); Faculdade de Ciências (da Saúde da Universidade da Beira Interior - 13,1\%, Médicas/Nova Medical School - 5,2\%); Escola de Medicina da Universidade do Minho (7,9\%); Departamento de Ciências Biomédicas e Medicina da Universidade do Algarve (8,3\%); e Instituto de Ciências Biomédicas de Abel Salazar (6,1\%).

\section{CONTACTO DIRECTO E FORMAÇÃO PRÉ-GRADUADA EM CUIDADOS PALIATIVOS}

A maioria (93\%) já tinha convivido em ambiente clínico com pessoas com doença incurável ou terminal, contudo 74\% nunca esteve numa Unidade de CP. A maioria teve acesso a aulas de CP (74\%), tendo sido elevada (87\%) a sua frequência (Tabela 1). Metade dos inquiridos teve aulas com duração total $<8$ horas, $e$ $23 \%$ tiveram mais de 8 horas de ensino (Tabela 2).

\section{ENSINO DE CUIDADOS PALIATIVOS E SUA IMPORTÂNCIA}

A maioria (97\%) estava consciente da necessidade de CP e do impacto das doenças crónicas na população portuguesa (77\%). Os participantes consideravam pertinente a inclusão 
Tabela 1: Ensino e contacto com os cuidados paliativos: Distribuição das respostas.

\begin{tabular}{|l|l|c|c|}
\hline \multicolumn{1}{|c|}{ Questões } & $\mathrm{n}=$ & Sim n (\%) & Não n (\%) \\
\hline Q1 - Já estiveste presente em alguma Unidade de Cuidados Paliativos? & 221 & $57(26 \%)$ & $164(74 \%)$ \\
\hline Q2 - Já conviveste em meio clínico com alguma pessoa com uma doença incurável ou terminal? & 221 & $205(93 \%)$ & $16(7 \%)$ \\
\hline Q3 - A tua faculdade inclui aulas de Cuidados Paliativos? & 221 & $164(74 \%)$ & $57(26 \%)$ \\
\hline Q4 - Se respondeste sim à Q3, frequentaste as aulas? & 164 & $143(87 \%)$ & $21(13 \%)$ \\
\hline
\end{tabular}

Tabela 2: Carga horária das aulas sobre cuidados paliativos: Distribuição das respostas ( $n=164)$.

\begin{tabular}{|c|c|c|c|c|c|c|}
\hline Questões & & & & & & \\
\hline \multirow[t]{2}{*}{ Se frequentaste as aulas, quantas horas foram? } & Horas & $\leq 4$ & $>4$ e $\leq 8$ & $>8$ e $\leq 18$ & $>18$ & Não sei \\
\hline & $\mathrm{n}(\%)$ & $30(18 \%)$ & $52(32 \%)$ & $21(13 \%)$ & $25(15 \%)$ & $36(22 \%)$ \\
\hline
\end{tabular}

da disciplina de CP na educação médica (96\%), estando familiarizados com o conceito de CP (75\%) e com os diferentes tipos de dor (51\%) (Tabela 3). No entanto, a maioria afirmava que a carga horária da disciplina de CP era insuficiente (64\%) e que a educação sobre CP no curso de medicina não correspondia ao conhecimento generalista que um médico devia possuir (52\%) (Tabela 3).

Constatou-se que apenas 37\% da amostra estava familiarizada com os sintomas de um doente terminal e com os sinais físicos de morte iminente (43\%), não estando confortável

Tabela 3: Avaliação e reconhecimento da importância do ensino de cuidados paliativos: Distribuição das respostas ( $\mathrm{n}=221)$.

\begin{tabular}{|c|c|c|c|c|c|c|}
\hline \multirow[b]{2}{*}{ Afirmações } & \multicolumn{6}{|c|}{ Grau de concordância } \\
\hline & $\begin{array}{l}\text { Concordo } \\
\text { Totalmente } \\
\mathrm{n}(\%)\end{array}$ & $\begin{array}{l}\text { Concordo } \\
\text { Bastante } \\
n(\%)\end{array}$ & $\begin{array}{l}\text { Concordo } \\
\text { Pouco } \\
\mathrm{n}(\%)\end{array}$ & $\begin{array}{l}\text { Discordo } \\
\text { Pouco } \\
\mathrm{n}(\%)\end{array}$ & $\begin{array}{l}\text { Discordo } \\
\text { Bastante } \\
\mathrm{n}(\%)\end{array}$ & $\begin{array}{l}\text { Discordo } \\
\text { Totalmente } \\
\mathrm{n}(\%)\end{array}$ \\
\hline $\begin{array}{l}\text { A1 - Estou familiarizado com o conceito de } \\
\text { cuidados paliativos. }\end{array}$ & 63 (29\%) & 101 (46\%) & $40(18 \%)$ & 10 (5\%) & 7 (3\%) & 0 \\
\hline $\begin{array}{l}\text { A2 - Estou familiarizado com os diferentes tipos } \\
\text { de dor. }\end{array}$ & 37 (17\%) & 75 (34\%) & 81 (37\%) & $8(4 \%)$ & $15(7 \%)$ & $5(2 \%)$ \\
\hline $\begin{array}{l}\text { A3 - Consigo imaginar-me a falar com um doente } \\
\text { terminal sobre morte. }\end{array}$ & 22 (10\%) & 39 (18\%) & 72 (33\%) & 21 (10\%) & 44 (20\%) & 23 (10\%) \\
\hline $\begin{array}{l}\text { A4 - Sinto-me familiarizado com os sintomas de } \\
\text { um doente em fase terminal. }\end{array}$ & 27 (12\%) & 55 (25\%) & 83 (38\%) & $19(9 \%)$ & 29 (13\%) & 8 (4\%) \\
\hline $\begin{array}{l}\text { A5 - Sinto-me familiarizado com os sinais físicos } \\
\text { de morte. }\end{array}$ & 29 (13\%) & 67 (30\%) & 67 (30\%) & 21 (10\%) & 31 (14\%) & $6(3 \%)$ \\
\hline $\begin{array}{l}\text { A6 - Sinto-me confortável com o processo de } \\
\text { comunicar más notícias a um doente/família. }\end{array}$ & 11 (5\%) & 35 (16\%) & $54(24 \%)$ & 23 (10\%) & 59 (27\%) & 39 (18\%) \\
\hline $\begin{array}{l}\text { A7 - Sinto-me consciente do impacto das doenças } \\
\text { crónicas e causadoras de longo sofrimento na } \\
\text { população. }\end{array}$ & 64 (29\%) & 105 (48\%) & 35 (16\%) & $8(4 \%)$ & 7 (3\%) & $2(1 \%)$ \\
\hline $\begin{array}{l}\text { A8 - Sinto-me consciente da necessidade de } \\
\text { Cuidados Paliativos na população. }\end{array}$ & 162 (73\%) & 52 (24\%) & $6(3 \%)$ & 0 & $1(0 \%)$ & 0 \\
\hline $\begin{array}{l}\text { A9 - Considero pertinente a inclusão da } \\
\text { disciplina de Cuidados Paliativos no currículo das } \\
\text { Faculdades de Medicina. }\end{array}$ & 176 (80\%) & 36 (16\%) & $9(4 \%)$ & 0 & 0 & 0 \\
\hline $\begin{array}{l}\text { A10 - A carga horária letiva da disciplina de } \\
\text { Cuidados Paliativos foi suficiente. }\end{array}$ & 11 (5\%) & $19(9 \%)$ & 31 (14\%) & 17 (8\%) & 56 (25\%) & 87 (39\%) \\
\hline $\begin{array}{l}\text { A11 - A educação em Cuidados Paliativos durante } \\
\text { o curso corresponde à formação necessária que } \\
\text { um médico generalista deveria ter. }\end{array}$ & 30 (14\%) & 30 (14\%) & 27 (12\%) & 18 (8\%) & 51 (23\%) & 65 (29\%) \\
\hline
\end{tabular}


com o processo de comunicação de más notícias (45\%). Quase um terço da amostra não se imaginava a falar com um doente terminal sobre a morte (Tabela 3).

\section{AFERIÇÃO DE NOÇÕES FUNDAMENTAIS SOBRE CUIDA- DOS PALIATIVOS}

Os inquiridos conheciam a filosofia dos CP, de modo geral, com mais de 63\% de tendências inequívocas e adequadas (Tabela 4). A maioria discordava indubitavelmente com as afirmações: a associação de CP com determinadas especialidades médicas (95\%); as dimensões espiritual, psicológica e social não constituírem uma preocupação médica (95\%); o sofrimento e a dor física serem sinónimos (91\%); nem todos os médicos necessitarem de conhecimentos em CP (89\%); a morte dever ser sempre combatida (83\%); Os CP serem compatíveis com um tratamento agressivo (79\%); os CP serem adequados apenas aquando de uma evidente deterioração clínica (78\%) (Tabela 4).

Os inquiridos concordavam que a equipa de saúde devia acompanhar a família, inclusive no luto (94\%), e que a família devia ser envolvida na atitude terapêutica do doente (93\%). Pese embora $67 \%$ da amostra considerasse que a medicina não deveria ser eminentemente curativa, cerca de 16\% discordou (Tabela 4).

Alguns inquiridos manifestaram alguma dificuldade em posicionar-se relativamente a: distanciamento emocional

Tabela 4: Conhecimentos teóricos sobre cuidados paliativos: Distribuição das respostas ( $\mathrm{n}=221$ ).

\begin{tabular}{|c|c|c|c|c|c|c|}
\hline \multirow[b]{2}{*}{ Afirmações } & \multicolumn{6}{|c|}{ Grau de concordância } \\
\hline & $\begin{array}{l}\text { Concordo } \\
\text { Totalmente } \\
\text { n (\%) }\end{array}$ & $\begin{array}{l}\text { Concordo } \\
\text { Bastante } \\
\text { n (\%) }\end{array}$ & $\begin{array}{l}\text { Concordo } \\
\text { Pouco } \\
\text { n (\%) }\end{array}$ & $\begin{array}{l}\text { Discordo } \\
\text { Pouco } \\
\text { n (\%) }\end{array}$ & $\begin{array}{l}\text { Discordo } \\
\text { Bastante } \\
\text { n (\%) }\end{array}$ & $\begin{array}{l}\text { Discordo } \\
\text { Totalmente } \\
\mathrm{n}(\%)\end{array}$ \\
\hline $\begin{array}{l}\text { A12 - Cuidados Paliativos são sinónimo de } \\
\text { Cuidados Terminais. }\end{array}$ & $1(0 \%)$ & $8(4 \%)$ & $25(11 \%)$ & $130(14 \%)$ & $83(38 \%)$ & $73(33 \%)$ \\
\hline $\begin{array}{l}\text { A14 - A filosofia dos Cuidados Paliativos é } \\
\text { compatível com um tratamento agressivo. }\end{array}$ & $3(1 \%)$ & $10(5 \%)$ & $10(5 \%)$ & $23(10 \%)$ & $49(22 \%)$ & $126(57 \%)$ \\
\hline $\begin{array}{l}\text { A15 - Para proporcionar Cuidados Paliativos } \\
\text { é necessário assegurar um distanciamento } \\
\text { emocional. }\end{array}$ & 2 (1\%) & 13 (6\%) & 29 (13\%) & 38 (17\%) & 67 (30\%) & 72 (33\%) \\
\hline A16 - Sofrimento e dor física são sinónimos. & $1(0 \%)$ & $2(1 \%)$ & $5(2 \%)$ & $14(6 \%)$ & $52(24 \%)$ & $147(67 \%)$ \\
\hline $\begin{array}{l}\text { A17 - Os conhecimentos de Cuidados Paliativos } \\
\text { dirigem-se apenas a médicos de especialidades } \\
\text { como medicina interna, medicina geral e familiar, } \\
\text { etc. }\end{array}$ & $4(2 \%)$ & 0 & 3 (1\%) & 5 (2\%) & 55 (25\%) & 154 (70\%) \\
\hline $\begin{array}{l}\text { A18 - Nem todos os médicos necessitam de } \\
\text { conhecimentos de Cuidados Paliativos, dado que } \\
\text { podem reencaminhar o doente para uma consulta } \\
\text { ou unidade de cuidados paliativos. }\end{array}$ & 0 & 0 & 10 (5\%) & 15 (7\%) & $33(24 \%)$ & $143(65 \%)$ \\
\hline $\begin{array}{l}\text { A19 - As dimensões espiritual, psicológica e } \\
\text { social do doente não devem ser preocupação do } \\
\text { médico, estando reservadas a outros profissionais } \\
\text { (psicólogos, assistentes sociais, enfermeiros e } \\
\text { outros). }\end{array}$ & $1(0 \%)$ & $1(0 \%)$ & $\begin{array}{c}1(0 \%) 1(0 \%) \\
3(1 \%) 6(3 \%) \\
33(15 \%) \\
177(80 \%)\end{array}$ & $6(3 \%)$ & $33(15 \%)$ & 177 (80\%) \\
\hline $\begin{array}{l}\text { A21 - A morte, sendo um resultado negativo da } \\
\text { perspetiva médica, deve ser sempre combatida, } \\
\text { usando todos os meios disponíveis. }\end{array}$ & 4 (2\%) & $6(3 \%)$ & $10(5 \%)$ & $18(8 \%)$ & $58(26 \%)$ & $125(57 \%)$ \\
\hline $\begin{array}{l}\text { A22 - No contexto de Cuidados Paliativos, a } \\
\text { família deve ser envolvida na atitude terapêutica } \\
\text { face ao doente. }\end{array}$ & $127(57 \%)$ & 79 (36\%) & $9(4 \%)$ & 5 (2\%) & 0 & $1(0 \%)$ \\
\hline $\begin{array}{l}\text { A23 - A equipa de Cuidados Paliativos deve } \\
\text { acompanhar e apoiar a família em todo o } \\
\text { processo, inclusive no luto. }\end{array}$ & 154 (70\%) & 53 (24\%) & $10(5 \%)$ & 1 (0\%) & $3(1 \%)$ & 0 \\
\hline
\end{tabular}


necessário para a prestação de CP (30\%); e CP como sinónimo de cuidados terminais (25\%) (Tabela 4).

\section{Discussão}

Dos 221 participantes, sendo 61\% estudantes, a maioria teve aulas de CP, com uma duração total $<8$ horas (50\%). Sobre a educação de CP, os inquiridos consideravam pertinente a inclusão da disciplina de CP na formação médica, com maior carga horária e que concedesse os conhecimentos necessários a qualquer médico generalista. A amostra opinou que devia existir mais formação sobre os sintomas de um doente terminal, os sinais físicos de morte iminente e a comunicação de más notícias. Os inquiridos revelaram um bom conhecimento sobre a filosofia dos CP e sentiam-se familiarizados com os principais conceitos da medicina paliativa. Apesar do contacto regular com doentes com necessidades paliativas, os estudantes não tinham acesso a um ambiente especializado em CP.

\section{COMPONENTE DEMOGRÁFICA}

O género e a idade dos inquiridos são compatíveis com o que ocorre nas EM e nos IFG em Portugal. O facto da maior parte dos inquiridos (45\%) provir da Faculdade de Medicina da Universidade de Lisboa deveu-se ao envolvimento e à divulgação institucional do inquérito junto dos estudantes.

\section{CONTACTO DIRETO E FORMAÇÃO PRÉ-GRADUADA EM CUIDADOS PALIATIVOS}

A maioria dos inquiridos (74\%) nunca esteve numa unidade de CP, uma prática contrastante com outras realidades, em que há uma dedicação horária exclusiva para interação com os doentes, como descrito numa EM Finlandesa. ${ }^{13}$ Este facto não pode ser totalmente atribuído a uma formação médica deficiente, atendendo ao número e distribuição geográfica das unidades de CP em Portugal. ${ }^{14}$

Pese embora a Lei n. ${ }^{0}$ 52/2012 tenha promulgado a criação da Rede Nacional de Cuidados Paliativos e decretado o direito de todos os cidadãos portugueses a aceder a CP, ${ }^{15}$ são conhecidos os problemas de referenciação, a heterogeneidade da cobertura nacional e a escassez de profissionais de saúde dedicados a CP. ${ }^{8}$ O "Observatório de Cuidados Paliativos" salienta os défices, as assimetrias e os entraves aos CP em Portugal. ${ }^{16}$ A situação é crítica em relação aos CP pediátricos. ${ }^{17}$

Positivamente, a maioria (93\%) da amostra já conviveu, em ambiente clínico, com indivíduos com doenças incuráveis ou terminais, sendo estes os casos mais referenciados para as unidades de CP. Assim, apesar da maioria dos estudantes não ter tido contacto com uma abordagem especializada em CP, durante a formação médica é garantido o contacto (quase inevitável) com doentes que necessitam desse tipo de cuidados, nos serviços hospitalares de medicina interna e/ou cirurgia geral.
A maioria dos inquiridos (74\%) teve acesso a aulas de CP, o que denota uma tendência crescente nos últimos anos nas EM. A propósito, a Resolução da Assembleia da República n. ${ }^{\circ}$ 5/2017 recomenda a implementação de formação pré e pós-graduada obrigatória em medicina paliativa, bem como a sua inclusão como especialidade reconhecida pela Ordem dos Médicos. ${ }^{18}$ A maioria dos estudantes (87\%) frequentou as aulas de CP. Metade dos inquiridos teve aulas com duração $\leq 8$ horas, menos do que se verifica na Suíça (25 horas de ensino obrigatório na Universidade de Basel) ou na Finlândia (53,5 horas de ensino obrigatório na Universidade de Tampere), países que têm investido no ensino de CP nas EM. ${ }^{13,19} \mathrm{~A}$ parcela de inquiridos com aulas de duração >18 horas (15\%) pode ser explicada pela frequência da disciplina opcional de CP nas EM, ou nos designados domínios verticais como ocorre na Universidade do Minho.

\section{ENSINO DE CUIDADOS PALIATIVOS E SUA IMPORTÂNCIA}

A maioria dos participantes estava familiarizada com o conceito de CP, uma tendência alinhada com outras realidades mundiais, como o Reino Unido, a Turquia e a Índia. 10,20,21

Mais de metade da amostra estava familiarizada com os diferentes tipos de dor. No entanto, é importante realçar que alguns estudos estrangeiros revelam que os estudantes evidenciam falta de conhecimentos e desconforto em lidar com a dor, assim como reportam um défice no ensino neste domínio. ${ }^{21-23}$ A dor é comum nos doentes seguidos por equipas de CP afectados por variadas patologias crónicas, em fases avançadas. ${ }^{23}$ É clara a necessidade de o controlo da dor fazer parte do leque de conhecimentos e competências de um médico. ${ }^{21}$ A Associação Internacional para o Estudo da Dor considera que as EM deveriam incluir o ensino da dor no seu currículo obrigatório, ${ }^{24}$ e o Programa Nacional de Controlo da Dor (da Direção- Geral de Saúde) refere a necessidade de melhorar a formação pré e pós-graduada na abordagem da dor. ${ }^{25} \mathrm{Um}$ estudo recente, acerca do ensino da dor crónica, analisou precisamente a perspetiva dos estudantes das oito EM e dos IFG em Portugal, concluindo que são necessárias mudanças no currículo das EM para que os futuros médicos desenvolvam competências e combatam o sofrimento "evitável" dos seus doentes.26 De facto, em Portugal, a aprendizagem da dor como "doença per se" não tem alocadas horas de ensino obrigatório em todas as EM, acabando o conhecimento por ser adquirido através do contacto com casos esporádicos, por interesse pessoal, ou posteriormente durante a experiência profissional. ${ }^{27}$

Em relação à identificação dos sintomas em CP e aos sinais físicos da iminência de morte, a generalidade das respostas indica que os inquiridos se sentiam familiarizados com os mesmos, não sendo possível, no entanto, observar uma tendência inequívoca. Na realidade, a Organização Mundial de Saúde preconiza a identificação precoce, avaliação adequada e tratamento rigoroso dos problemas físicos, psicossociais e espirituais dos doentes em CP. ${ }^{28,29}$ 
Os participantes denotaram um desconforto na relação com o doente, mais claro na comunicação de más notícias. Quase um terço da amostra não imaginava uma conversa sobre a morte com um doente terminal. Tal indica que durante a educação médica há carências ao nível da aprendizagem e da prática da relação médico-doente terminal. Outros estudos suportam a preocupação acerca da qualidade de comunicação de más notícias por parte de médicos e estudantes de medicina, que se consideram mal preparados para o efeito após o término da educação pré-graduada. ${ }^{10,21,30-32} \mathrm{~A}$ falta de preparação em CP dos estudantes, relativamente à comunicação com os doentes em fase paliativa, está documentada. ${ }^{21,33}$

A prevalência crescente de doenças crónicas está intimamente relacionada com a prática de $\mathrm{CP}$, sejam os doentes oncológicos ou não. ${ }^{33}$ Os inquiridos, em relação a estas doenças, estão conscientes do impacto das mesmas na população, sendo causadoras de sofrimento de longa duração, da necessidade de CP e da importância da disciplina de CP. Anualmente, cerca de $71 \%$ de todas as mortes são causadas por doenças crónicas. ${ }^{34}$ Cerca de sete em cada dez mortes, em Portugal, são causadas por doenças que beneficiavam de CP.35 É sabido que a maioria dos adultos que necessitam de $\mathrm{CP}$ tem doenças com agravamento progressivo, como cardiovasculares (38,5\%), cancro (34\%), respiratórias (10,3\%), SIDA (5,7\%) e diabetes mellitus (4,6\%). ${ }^{36}$ Este número de pessoas que necessita de CP terá tendência para aumentar futuramente. ${ }^{37}$

A maioria era da opinião que a carga horária da disciplina de CP foi insuficiente durante o curso. Através da consulta dos planos de estudo das EM portuguesas, disponíveis nos respetivos sítios eletrónicos oficiais (março de 2019), constatou-se que todas abordam o tema dos $\mathrm{CP}$, mas a maioria fá-lo em currículo optativo. A educação insuficiente em CP é também uma preocupação partilhada por estudantes de universidades estrangeiras, de contextos distintos, nomeadamente do Reino Unido, Áustria, Omã ou Turquia, constatando-se uma ausência de correspondência entre a necessidade de formação em CP (reconhecida pelos estudantes) e a oferta curricular vigente. 21,32,38,39

O termo composto "educação insuficiente" remete para uma preparação incompleta em CP para o exercício da profissão, noção comprovada pelas respostas obtidas em A11 que, apesar de uma distribuição dispersa, tendem mais para "Discordo Totalmente" (29\%). Esta distribuição é compreensível, dados: o grau variável de conhecimentos de CP, as possiveis diferentes opiniões acerca do adequado funcionamento e meIhoria dos CP, e a opinião acerca da extensão de conhecimento de CP que um médico generalista deveria possuir.

Em Portugal, a educação médica em CP situa-se numa posição inferior ao percentil 50 (afastado de países como Israel, Noruega e o Reino Unido), segundo uma investigação promovida pela Associação Europeia de CP. ${ }^{40}$ Nesta, o objeto de estudo foram as universidades europeias e foi tido em conta: o número de EM de cada país, a existência da disciplina de CP, e o seu caráter opcional ou obrigatório.40 É importante, no entanto, não ignorar o trajeto positivo que as EM têm vindo a percorrer no sentido de melhorar a formação médica pré-graduada em CP, mormente desde o ano letivo 2016/2017.

A educação adequada em CP aporta vantagens significativas para os estudantes e os jovens médicos. Um estudo espanhol - que analisou qualitativamente o impacto de um curso opcional de CP nos estudantes, durante 4 anos, - concluiu que: os alunos são ajudados a tornarem-se e a agirem como médicos; os benefícios de uma visão holística sobre o doente são identificados, assim como a importância de envolver a sua família; os CP possibilitam a abertura de um novo campo de conhecimento; a reflexão dos estudantes acerca do seu desenvolvimento pessoal é estimulada e são aprofundadas as capacidades humanísticas; por último, o contacto prático com os doentes é essencial. ${ }^{41}$ Estas evidências aumentam a confiança num avanço curricular na área da medicina paliativa, desejado tanto pelos órgãos de ensino e da saúde, quanto por toda a comunidade de estudantes de medicina.

\section{AFERIÇÃO DE NOÇÕES FUNDAMENTAIS SOBRE CUIDA- DOS PALIATIVOS}

A clara tendência de resposta foi, em todas as afirmações da Tabela 4, concordante com a filosofia atual dos CP. ${ }^{3}$ As oito afirmações, que apresentam uma das seis hipóteses com a maioria de respostas, revelam que os inquiridos consideravam que os CP: não são compatíveis com um tratamento agressivo; estão direcionados para os vários tipos de dor e para o sofrimento, nunca esquecendo a sua diferença; são praticados aceitando a morte como um processo natural; estão atentos à família, acompanhando-a e apoiando-a., 3,42

Apesar da literatura evidenciar que o conhecimento dos estudantes sobre CP é limitado, devendo ser privilegiada a meIhoria do ensino nesta matéria, ${ }^{21,43-47}$ a maioria dos participantes (89\%) reconheceu a necessidade desse conhecimento.

Os estudantes de medicina não estão preparados para cuidarem de doentes com doenças terminais e em fim de vida, especialmente no acolhimento das suas necessidades psicossociais e espirituais. ${ }^{43}$ Todavia, a maioria dos inquiridos (95\%) assume as dimensões espiritual, psicológica e social como parte da preocupação médica.

Os inquiridos sabem que os conhecimentos em CP são transversais a todos os médicos, através de uma abordagem paliativa que encara a pessoa doente como um todo. Se os médicos especialistas em CP tomassem a seu cargo todos os casos em fase paliativa, os médicos generalistas e outros especialistas poderiam começar a desresponsabilizar-se no que toca ao controlo básico de sintomas e ao apoio psicossocial da díade doente/família. Além disto, naturalmente que não existem e nunca existirão especialistas em CP suficientes para cobrir todas as necessidades de um país. ${ }^{20,33}$ 
Nas outras quatro afirmações que constam da Tabela 4, nomeadamente A12, A13, A15 e A20, apesar de haver alguma dispersão, a direção das respostas foi correta. Estas variações podem dever-se a afirmações que suscitam mais dúvidas: a confusão entre CP e Cuidados Terminais (A12) que, apesar de se sobreporem, não se limitam à terminalidade da vida; a dúvida de os CP também se aplicarem a doentes que não apresentam uma clara trajetória de deterioração (A13); a relação emocional adequada a desenvolver com o doente (A15); finalmente, o intuito apenas curativo versus o evitamento da cronicidade (A20).

No seu conjunto, este estudo apresenta uma amostra de estudantes e IFG que têm em mente os princípios básicos dos CP, subsistindo, no entanto, algumas dúvidas que revelam um deficiente contacto com a medicina paliativa e um escasso número de horas de currículo obrigatório em $\mathrm{CP}$. De facto, o contacto prático - em falta no ensino de CP - é essencial, permitindo não só a melhoria das competências clínicas como a melhoria da relação médico-doente. ${ }^{41}$ Os estudantes que frequentaram um curso de CP mostraram-se surpresos com a visão humana e holística providenciada pelo curso, sentiam-se mais preparados para a prática clínica, e afirmavam que a aprendizagem em CP é útil e aplicável a qualquer tipologia de doente..$^{35,48}$

As recomendações internacionais e nacionais fomentam o investimento em CP na formação de futuros médicos, visando a capacitação destes, para melhor responderem às necessidades das pessoas com doenças crónicas, avançadas. , $49,50^{-5}$ Portugal tem feito um esforço neste sentido, mas há ainda uma oportunidade de crescimento, de modo a normalizar a medicina paliativa como parte integrante de um ensino médico de excelência.

\section{LIMITAÇÕES DO ESTUDO}

Este estudo tem várias limitações.

Não se pode garantir a representatividade dos estudantes e IFG.

O preenchimento do questionário foi voluntário, não resultando numa amostra aleatória simples, o que pode constituir uma fonte de erro.

No que diz respeito ao pedido de divulgação do questionário eletrónico, este foi assegurado sobretudo pelas Associações de Estudantes das oito EM. Apenas a Direção da Faculdade de Medicina da Universidade de Lisboa deferiu o pedido e, através do seu Gabinete de Gestão Curricular, difundiu o inquérito pelos estudantes. Em relação às outras EM, as formalidades exigidas pelas respetivas Direções não puderam ser cumpridas no curto intervalo em que o estudo decorreu.

Houve ainda outras limitações. Não foi realizada uma análise da validade do questionário como instrumento de medição. Não foi realizada uma distinção entre o currículo obrigatório e o opcional em $\mathrm{CP}$, que tem variações nas oito
EM, um aspeto que podia ser diferenciador ao analisar as respostas. Não foi questionada a extensão de conhecimento de $\mathrm{CP}$ que um médico generalista deveria ter.

\section{Conclusão}

Existem três principais conclusões deste estudo.

A primeira. A educação sobre CP é pertinente, devendo existir uma disciplina de CP no curso de medicina, com maior carga horária, que conceda os conhecimentos necessários a qualquer médico generalista. É necessária mais formação sobre os sintomas de um doente terminal, os sinais físicos de morte iminente, e a comunicação de más notícias.

A segunda. Existe um bom conhecimento da filosofia dos $\mathrm{CP}$, existindo familiaridade com os principais conceitos da medicina paliativa. Destacam-se os seguintes aspetos: todos os médicos necessitam de conhecimentos em CP, independentemente da especialidade médica; a medicina não deve ser apenas curativa; a preocupação médica agrega as dimensões espiritual, psicológica e social do doente/família, para além do sofrimento físico; o envolvimento da família nas decisões terapêuticas; o acompanhamento da família, inclusive no luto.

A terceira. O ensino de CP ao longo do curso de medicina é maioritariamente de cariz teórico. Apesar do contacto com os doentes que necessitam de $\mathrm{CP}$, os estudantes não têm acesso a um ambiente especializado em CP, o que não contribui para a total compreensão da praxis da medicina paliativa, nem para o incentivo ao exercício da abordagem paliativa num futuro profissional.

\section{Responsabilidades Éticas}

Conflitos de Interesse: Os autores declaram a inexistência de conflitos de interesse na realização do presente trabalho.

Fontes de Financiamento: Não existiram fontes externas de financiamento para a realização deste artigo.

Confidencialidade dos Dados: Os autores declaram ter seguido os protocolos da sua instituição acerca da publicação dos dados de doentes.

Proteção de Pessoas e Animais: Os autores declaram que os procedimentos seguidos estavam de acordo com os regulamentos estabelecidos pelos responsáveis da Comissão de Investigação Clínica e Ética e de acordo com a Declaração de Helsínquia da Associação Médica Mundial. Proveniência e Revisão por Pares: Não comissionado; revisão externa por pares.

\section{Ethical Disclosures}

Conflicts of interest: The authors have no conflicts of interest to declare. Financing Support: This work has not received any contribution, grant or scholarship

Confidentiality of Data: The authors declare that they have followed the protocols of their work center on the publication of data from patients.

Protection of Human and Animal Subjects: The authors declare that the procedures followed were in accordance with the regulations of the relevant clinical research ethics committee and with those of the Code of 


\author{
Ethics of the World Medical Association (Declaration of Helsinki). \\ Provenance and Peer Review: Not commissioned; externally peer re- \\ viewed.
}

(c) Autor (es) (ou seu (s) empregador (es)) e Revista SPMI 2021. Reutilização permitida de acordo com CC BY-NC. Nenhuma reutilização comercial. (C) Author(s) (or their employer(s)) and SPMl Journal 2021. Re-use permitted under CC BY-NC. No commercial re-use.

\section{Correspondence / Correspondência:}

Pedro Frazão - pedroruifrazao@gmail.com

Médico, Medicina Interna, Hospital Ortopédico Sant'iago do Outão, Centro Hospitalar de Setúbal, Setúbal, Portugal

Outão Senhor da Anunciada, 2900-180 Setúbal

\section{Received / Recebido: 08/09/2020}

Accepted / Aceite: 21/10/2020

\section{Publicado / Published: 15 de março de 2021}

\section{REFERÊNCIAS}

1. Council of Europe. Parliamentary Assembly. Committee on Social Affairs, Health and Sustainable Development. The provision of palliative care in Europe. Report Doc. 14657; 2018 [consultado 2020 Jul 17]. Disponível em: http://assembly.coe.int/nw/xml/XRef/Xref-XML2HTML-en. asp? fileid $=25057$ \&lang=en.

2. Gamondi C, Larkin P, Payne S. Core competencies in palliative care: an EAPC white paper on palliative care education: part 1. Eur J Palliat Care. 2013;20:86-91.

3. World Health Organization. World Palliative Care Alliance. Global Atlas of Palliative Care at the End of Life; 2014 [consultado 2020 Jul 17]. Disponível em: https://www.who.int/nmh/Global_Atlas_of_Palliative_Care.pdf.

4. Neto IG. Cuidados Paliativos: Princípios e conceitos fundamentais. In: Barbosa A, Pina PR, Tavares F, Neto IG, editores. Manual de Cuidados Paliativos. 3. ${ }^{a}$ ed. Lisboa: Faculdade de Medicina da Universidade de Lisboa; 2016. p. 1-22.

5. Comissão Nacional de Cuidados Paliativos. Plano Estratégico para o Desenvolvimento dos Cuidados Paliativos Biénio 2019-2020; 2019 [consultado 2020 Jul 17]. Disponível em: https://www.sns.gov.pt/wp-content/ uploads/2019/04/PEDCP-2019-2020-versao-final-10.02.2019.pdf.

6. Council of Europe. Recommendation Rec (2003) 24 of the Committee of Ministers to Member States on the Organisation of Palliative Care; 2003 [consultado 2020 Jul 17]. Disponível em: https://www.coe.int/t/dg3/health/ Source/Rec(2003)24_en.pdf.

7. Morais Al. Cuidados Continuados e Paliativos (CCP): um estudo descritivo sobre o conhecimento de estudantes de áreas da saúde. [Tese de Mestrado em Psicologia Clínica e da Saúde]. Gandra: Instituto Superior de Ciências da Saúde do Norte; 2013 [consultado 2020 Jul 17]. Disponível em: https://repositorio.cespu.pt/handle/20.500.11816/282

8. Observatório Português dos Sistemas de Saúde. Relatório Primavera 2017. Viver em tempos incertos: sustentabilidade e equidade na saúde; 2017 [consultado 2020 Jul 17]. Disponível em: http://opss.pt/relatorios/ relatorio-primavera-2017/.

9. Comissão Nacional de Cuidados Paliativos. Plano Estratégico para o Desenvolvimento dos Cuidados Paliativos Biénio 2017-2018; 2016 [consultado 2020 Jul 17]. https://www.sns.gov.pt/wp-content/uploads/2016/09/ Plano-Estrat\%C3\%A9gico-CP_2017-2018-1.pdf.

10. Bharadwaj $P$, Vidyasagar MS, Kakria A, Alam UA. Survey of palliative care concepts among medical interns in India. J Palliat Med. 2007;10:654-7.

11. Yamamoto R, Kizawa Y, Nakazawa Y, Morita T. The Palliative Care Knowledge Questionnaire for PEACE: reliability and validity of an instrument to measure palliative care knowledge among physicians. J Palliat Med. 2013;16:1423-8
12. Chover-Sierra E, Martínez-Sabater A, Lapeña-Moñux YR. An instrument to measure nurses knowledge in palliative care: validation of the Spanish version of Palliative Care Quiz for Nurses. PLoS One. 2017;12:e0180622.

13. Lehto JT, Hakkarainen K, Kellokumpu-Lehtinen PL, Saarto T. Undergraduate curriculum in palliative medicine at Tampere University increases students' knowledge. BMC Palliat Care. 2017;16:13.

14. Ministério da Saúde, Serviço Nacional de Saúde. Equipas de Cuidados Paliativos. Unidades de Cuidados Paliativos (atualização 15-06-2020) [consultado 2020 Jul 17]. Disponível em: https://www.sns.gov.pt/wp-content/ uploads/2020/05/UCP_05052020.pdf.

15. Assembleia da República. Lei $n^{\circ}$ 52/2012. Diário da República $n$. $^{\circ}$ 172/2012, Série I de 2012-09-05. p. 5119-24 [consultado 2020 Jul 17]. Disponível em: https://data.dre.pt/eli/lei/52/2012/09/05/p/dre/pt/html.

16. Observatório Português de Cuidados Paliativos. Relatório Outono 2019. Secção I. Cobertura e caracterização das equipas e profissionais das equipas de cuidados paliativos. Lisboa, Portugal: Universidade Católica Editora; 2020. p. 5-43 [consultado 2020 Jul 17]. Disponível em: http:// www.uceditora.ucp.pt/resources/Documentos/UCEditora/PDF\%20Livros/ OPCP\%2ORELAT\%C3\%93RIO\%20OUTONO\%202019.pdf.

17. Lacerda AF, Gomes B. Trends in cause and place of death for children in Portugal (a European country with no Paediatric palliative care) during 1987-2011: a population-based study. BMC Pediatr. 2017;17:215.

18. Assembleia da República. Resolução da Assembleia da República n. ${ }^{\circ}$ 5/2017. Diário da República n. $3 / 2017$, Série I de 2017-0104 [consultado 2020 Jul 17]. Disponível em: https://data.dre.pt/eli/ resolassrep/5/2017/01/04/p/dre/pt/html.

19. Eychmüller S, Forster M, Gudat H, Lütolf UM, Borasio GD. Undergraduate palliative care teaching in Swiss medical faculties: a nationwide survey and improved learning objectives. BMC Med Educ. 2015;15:213-20.

20. Quill TE, Abernethy AP. Generalist plus specialist palliative care - creating a more sustainable model. N Engl J Med 2013;368:1173-5.

21. Eyigor S. Fifth-year medical students' knowledge of palliative care and their views on the subject. J Palliat Med. 2013;8:941-6.

22. Dalpai D, Mendes FF, Asmar JA, Carvalho PL, Loro FL, Branco A. Dor e cuidados paliativos: o conhecimento dos estudantes de medicina e as lacunas da graduação. Rev Dor. 2017;18:307-10.

23. Tran UE, Kircher J, Jaggi P, Lai H, Hillier T, Ali S. Medical students' perspectives of their clinical comfort and curriculum for acute pain management. J Pain Res. 2018;11:1479-88.

24. Kopf A, Treede RD, Graven-Nielsen T, Cadavid A, Rathmell J. IASP Curriculum Outline on pain for medicine; 2018 [consultado em 2020 Jul 17]. Disponível em: https://www.iasp-pain.org/Education/CurriculumDetail. aspx?ItemNumber=729.

25. Direção Geral de Saúde. Circular Normativa n¹1. Programa Nacional de Controlo da Dor; 2008 [consultado em 2020 Jul 17]. Disponível em: https://www.dgs.pt/areas-em-destaque/plano-nacional-de-saude/programas-nacionais/programa-nacional-de-controlo-da-dor.aspx.

26. Cristóvão I, Reis-Pina P. Chronic pain education in Portugal: perspectives from medical students and interns. Acta Med Port. 2019;32:338-47.

27. Centro Nacional de Observação em Dor - Observador. Estado da arte do ensino da dor em Portugal; 2010 [consultado em 2020 Jul 17]. Disponível em: https://www.dgs.pt/documentos-e-publicacoes/estado-da-arte-do-ensino-da-dor-em-portugal-relatorio-final.aspx.

28. World Health Organization. WHO Definition of palliative care [homepage na Internet] [consultado 2020 Jul 17]. Disponível em: http://www.who.int/ cancer/palliative/definition/en/.

29. Associação Nacional de Cuidados Paliativos. Organização de serviços em cuidados paliativos - Recomendações da ANCP; 2006 [consultado 2020 Jul 17]. Disponível em: https://www.apcp.com.pt/uploads/Recomendacoes_Organizacao_de_Servicos.pdf.

30. Coutinho F, Ramessur A. An overview of teaching communication of bad news in medical school: should a lecture be adequate to address the topic? Acta Med Port. 2016;29:826-31.

31. Alelwani SM, Ahmed YA. Medical training for communication of bad news: A literature review. J Educ Health Promot. 2014;3:51.

32. Lee AY, Carlon B, Ramsay R, Thirukkumaran T. Integrating exposure to palliative care in an undergraduate medical curriculum: student perspectives and strategies. Int J Med Educ. 2017;8:151-2.

33. Lima MS. Formação em cuidados paliativos. Influência na vida profissional. Dissertação de Mestrado em Cuidados Paliativos. Faculdade de 
Medicina da Universidade do Porto; 2018 [consultado 2020 Jul 17]. Dispo nível em: https://sigarra.up.pt/fmup/pt/pub_geral.pub_view?pi_pub_base_ id $=242882$.

34. World Health Organization. Fact Sheets - Noncommunicable Diseases 2018 [consultado 2020 Jul 17]. Disponível em: https://www.who.int/news-room/fact-sheets/detail/noncommunicable-diseases.

35. Gomes B, Pinheiro MJ, Lopes S, Brito M, Sarmento VP, Ferreira PL, et al Risk factors for hospital death in conditions needing palliative care: Nationwide population-based death certificate study. Palliat Med. 2018;32:891-901.

36. World Health Organization. Fact Sheets - Palliative Care; 2018. [consultado 2020 Jul 17]. Disponível em: https://www.who.int/news-room/fact-sheets/ detail/palliative-care

37. Stjernswärd J, Foley KM, Ferris FD. The public health strategy for palliative care. J Pain Symptom Manage. 2007;33:486-93.

38. Pohl G, Marosi C, Dieckmann K, Goldner G, Elandt K, Hassler M, et. al Survey of palliative care concepts among medical students and interns in Austria: A comparison of the old and the new curriculum of the Medical University of Vienna. Palliat Care Res Treat. 2008;2:1-7.

39. Jahan F, Shibli HAS, Qatan RS, Kharusi AAA. Perception of undergraduate medical students in clinical years regarding palliative care. Middle East $J$ Age Ageing. 2010;7:22-31.

40. Carrasco JM, Lynch TJ, Garralda E, Woitha K, Elsner F, Filbet M, et al Palliative care medical education in European universities: a descriptive study and numerical scoring system proposal for assessing educational development. J Pain Symptom Manage. 2015;50:516-23.

41. Centeno C, Ballesteros M, Carrasco JM, Arantzamendi M. Does palliative care education matter to medical students? The experience of attending an undergraduate course in palliative care. BMJ Support Palliat Care. 2016;6:128-34.

42. Hartogh GD. Suffering and dying well: on the proper aim of palliative care. Med Health Care Philos. 2017;20:413-24.
43. Weber M, Schmiedel S, Nauck F, Alt-Epping B. Knowledge and attitude of final-year medical students in Germany towards palliative care - an interinstitutional questionnaire-based study. BMC Palliat Care. 2011;10:19.

44. Pinheiro TR. Avaliação do grau de conhecimento sobre cuidados paliativos e dor dos estudantes de medicina do quinto e sextos anos. Mundo Saúde. 2010;34:320-6.

45. Lemos CF, Barros GS, Melo NC, Amorim FF, Santana AN. Avaliação do conhecimento em cuidados paliativos em estudantes durante o curso de medicina. Rev Bras Educ Med. 2017;41:278-82.

46. Hesselink BA, Pasman HR, Wal G, Soethout MB, Onwuteaka-Philipsen $\mathrm{BD}$. Education on end-of-life care in the medical curriculum: students' opinions and knowledge. J Palliat Med. 2010;4:381-7.

47. Pandey S, Gaire D, Dhakal S, Jaishwal N, Kharal PM, Vaidya P. Perception of palliative care among medical students in a teaching hospital. J Nepal Med Assoc. 2015;53:113-7.

48. Orlander JD, Fincke BG, Hermanns D, Johnson GA. Medical residents' first clearly remembered experiences of giving bad news. J Gen Intern Med. 2002;17:825-31.

49. European Association for Palliative Care. Recommendations of the European Association for Palliative Care (EAPC) for the development of undergraduate curricula in palliative medicine at European Medical Schools; 2013 [consultado 2020 Jul 17]. Disponível em: https://www.eapcnet.eu/ Portals/O/adam/Content/70PKe-5EvkmDsBh1s1UWPA/Text/Recommendations\%20of\%20the\%20EAPC\%20for\%20the\%20development\%20 of\%20undergraduate\%20curricula\%20in\%20palliative\%20medicine\%20 at\%20European\%20Medical\%20Schools.pdf

50. European Federation of Older Persons. Making palliative care a priority topic on the European health agenda: and recommendations for the development of palliative care in Europe; 2004 [consultado 2020 Jul 17] Disponível em: https://www.apcp.com.pt/uploads/Cons.Europa_EURAG_ PalliativeCareProject_2004.pdf. 